\title{
El tamizaje de cáncer de mama con mamografía reduce la mortalidad específica en mujeres de 40 a 48 años
}

\author{
Breast cancer screening with mammography reduces specific mortality in women aged 40-48 years
}

\section{Comentado de:}

Duffy SW, et al. Lancet Oncol 2020; 21:1165-72.PMID: $32800099^{1}$

\section{Objetivo}

Evaluar el efecto del tamizaje anual con mamografía para reducir la mortalidad por cáncer de mama en comparación con la ausencia de cribado, en mujeres de 40 a 48 años.

\section{Diseño y lugar}

Ensayo clínico controlado y aleatorizado realizado en Gran Bretaña.

\section{Participantes}

Se identificaron mujeres de 39 a 41 años de edad tomando las listas de pacientes de médicos de familia. Se envió una carta para invitar a las pacientes del grupo intervención. Las mujeres del grupo control recibieron la atención médica habitual sin estar al tanto del estudio.

\section{Intervención}

Se asignaron mujeres al azar y estratificando por centro de atención primaria en una proporción 1:2. El grupo intervención realizó tamizaje anual con mamografía convencional desde el año de inclusión hasta que las mujeres cumplieran 48 años y el grupo control no realizó tamizaje hasta la edad de 50 años, momento en que todas las mujeres reciben una invitación para realizar esta práctica cada 3 años.

\section{Medición de resultados principales}

El desenlace primario fue la mortalidad por cáncer de mama diagnosticado en el período de la intervención (luego de la aleatorización hasta la primera invitación a los 50 años de edad).

Los desenlaces secundarios fueron la mortalidad por cáncer de mama diagnosticado luego de la aleatorización hasta el final de la recolección de los datos, la mortalidad por otras causas, la mortalidad global y la incidencia de cáncer de mama. El análisis primario fue por intención de tratar.

\section{Resultados Principales}

El enrolamiento se realizó desde 1990 hasta 1997. La mediana de seguimiento fue de 22,8 años (intervalo intercuartilo 21,8 a 24,0$)$. De las 160.836 mujeres incluidas en el estudio, 150.909 (93,8\%), $38.988(24,2 \%)$ y $9.605(5,9 \%)$ completaron 20,24 y 25 años de seguimiento, respectivamente. Las pérdidas fueron proporcionalmente similares en los grupos intervención y control.

La diferencia absoluta fue de $-0,6$ muertes por cada 1.000 mujeres invitadas al tamizaje (intervalo de confianza [IC] del $95 \%$ $-1,3$ a 0,1$)$ y se mantuvo constante a lo largo del seguimiento. Durante el período de intervención, 7.893 (18,1\%) de 43.709 mujeres que se realizaron mamografías de tamizaje tuvieron al menos un resultado falso positivo. En la Tabla 1 se resumen los principales resultados del estudio.

Tabla 1. Efecto del tamizaje anual con mamografía en mujeres de 40 a 50 años

\begin{tabular}{|c|c|c|c|}
\hline Desenlace & $\begin{array}{l}\text { Grupo Intervención } \\
\text { (personas-año de seguimiento) }\end{array}$ & $\begin{array}{l}\text { Grupo Control } \\
\text { (personas-año de seguimiento) }\end{array}$ & $\begin{array}{l}\text { Riesgo Relativo } \\
\text { (IC } 95 \% \text { ) }\end{array}$ \\
\hline $\begin{array}{l}\text { Mortalidad por cáncer de mama } \\
\text { (10 años de seguimiento) }\end{array}$ & $83(532.729)$ & $219(1.058 .236)$ & $0,75(0,58$ a 0,97$)$ \\
\hline $\begin{array}{l}\text { Mortalidad por cáncer de mama } \\
\text { (más de } 10 \text { años hasta final del seguimiento) }\end{array}$ & $126(668.281)$ & $255(1.326 .770)$ & $0,98(0,79$ a 1,22$)$ \\
\hline $\begin{array}{l}\text { Mortalidad por cáncer de mama } \\
\text { (total) }\end{array}$ & $209(1.201 .010)$ & $474(2.385 .006)$ & $0,88(0,74$ a 1,03$)$ \\
\hline $\begin{array}{l}\text { Mortalidad por todas las causas (al final del } \\
\text { seguimiento) }\end{array}$ & 3.507 (no informado) & 6.932 (no informado) & $1,01(0,96$ a 1,05$)$ \\
\hline $\begin{array}{l}\text { Incidencia global de cáncer de mama (al final } \\
\text { del seguimiento) }\end{array}$ & $2.617(1.174 .649)$ & $5.260(2.334 .516)$ & $0,99(0,94$ a 1,04$)$ \\
\hline $\begin{array}{l}\text { Incidencia cánceres de mama invasivos (al } \\
\text { final del seguimiento) }\end{array}$ & $2.288(1.177 .990)$ & $4.640(2.339 .852)$ & $0,98(0,93$ a 1,03$)$ \\
\hline $\begin{array}{l}\text { Incidencia cánceres de mama in-situ (perío- } \\
\text { do intervención) }\end{array}$ & $118(573.221)$ & $103(1.137 .432)$ & $2,27(1,75$ a 2,95$)$ \\
\hline $\begin{array}{l}\text { Incidencia cánceres de mama in-situ (al final } \\
\text { del seguimiento) }\end{array}$ & $329(1.195 .224)$ & $620(2.375 .349)$ & $1,05(0,92$ a 1,20$)$ \\
\hline
\end{tabular}

\section{Conclusiones}

La mamografía anual a partir de los 40 o 41 años de edad se asoció con una reducción relativa de la mortalidad por cáncer de mama con respecto a su iniciación a partir de los 50 años. Esta diferencia se atenuó después de 10 años de seguimiento, aunque la reducción absoluta se mantuvo constante.

Reducir el límite inferior de edad para la detección de 50 a 40 años podría reducir potencialmente la mortalidad por cáncer de mama. 
Fuente de financiamiento/Conflicto de interés de los autores: El estudio fue financiado por el Instituto Nacional de Investigación en Salud. Los autores recibieron becas del Instituto Nacional para la Evaluación de Tecnologías de Investigación en Salud, la Sociedad Estadounidense del Cán- cer, la Investigación del Cáncer del Reino Unido, el Departamento de Salud, el Consejo de Investigación Médica y el Instituto Nacional del Cáncer de los EE.UU. Uno de los autores trabaja en una empresa privada de diagnóstico de cáncer (GRAIL).

\section{Comentario}

Los resultados de este ensayo clínico luego de 23 años de seguimiento, son similares a los publicados en $2015^{2}$ y fueron incluidos en diversos meta-análisis ${ }^{3-5}$. Se estima que de cada 10.000 mujeres de 40 a 49 años de edad que se realizan el tamizaje a lo largo de 10 años, tres evitarán morir por cáncer de mama, mientras que 6.130 tendrán un resultado falso positivo, 700 se realizarán una biopsia innecesaria y 28 recibirán un sobrediagnóstico (un diagnóstico de cáncer que nunca hubiese generado un problema de salud si no era detectado) ${ }^{6,7}$. Sin embargo, como lo señala este estudio y los meta-análisis citados, el tamizaje del cáncer de mama no demostró reducir la mortalidad global en este grupo etario.

La evidencia sobre la efectividad de esta práctica presenta ciertas controversias y limitaciones ${ }^{5-8}$. Por un lado, se estipula que las técnicas mamográficas más nuevas (no probadas en los ensayos clínicos realizados hace más de 30 años) resultarían en una mayor reducción de la mortalidad y menos falsos positivos, aunque es poco probable que se repitan los ensayos para confirmar esta hipótesis. Por otro lado, los estudios observacionales sugieren que el tamizaje desempeña un papel menor en la reducción de la mortalidad, y que ésta probablemente sea el resultado de los avances en los tratamientos y la concientización sobre la enfermedad, que logra que las mujeres sintomáticas consulten precozmente $591011,12$.

La magnitud del sobrediagnóstico es otro tema en discusión. En mujeres de 40 a 49 años de edad, el único ensayo clínico que incluyó un grupo control que nunca realizó tamizaje mostró que este fenómeno se presenta entre el $12,4 \%$ y el 29,4\% de las participantes ${ }^{13}$.

En base a esta controversia, el Instituto Nacional del Cáncer de Argentina -que también evaluó este ensayo para elaborar sus recomendaciones- sugiere realizar el tamizaje únicamente en mujeres de 50 a 70 años, mientras que, en mujeres de 40 a 50 años, sugiere evaluar cada caso de manera individual ${ }^{14}$. Cada vez más las guías de práctica clínica recomiendan que la decisión de realizar o no el tamizaje sea tomada por los profesionales de la salud en conjunto con las pacientes, informándoles tanto sobre los beneficios como los daños del estudio y priorizando lo más importante para ellas, acorde a sus valores y preferencias ${ }^{6}$. Existen herramientas desarrolladas localmente para ayudar a profesionales de la salud a presentar información balanceada y basada en evidencia científica que apoye la toma de decisiones compartidas con las pacientes durante la consulta ${ }^{15}$.

\section{Conclusiones de la comentadora}

Iniciar el tamizaje a partir de los 40 años de edad puede reducir la mortalidad por cáncer de mama, así como también aumentar los resultados falsos positivos, los sobrediagnósticos, las biopsias y los tratamientos innecesarios. Se aconseja emplear herramientas específicas que ayudan a la toma de decisiones en las consultas con las pacientes para definir la realización de esta práctica.

Paula Riganti [ Servicio de Medicina Familiar y Comunitaria, Hospital Italiano de Buenos Aires. paula.riganti@hospitalitaliano.org.ar ]

Riganti P El tamizaje de cáncer de mama con mamografía reduce la mortalidad específica en mujeres de 40 a 48 años. Evid Actual Pract Ambul. $2021 ; 24(1)$ :e002110. Comentado de: Duffy SW, et al. Effect of mammographic screening from age 40 years on breast cancer mortality (UK Age trial): final results of a randomised, controlled trial. Lancet Oncol. 2020;21(9):1165-1172. PMID: 32800099

\section{Referencias}

1. Duffy SW, Vulkan D, Cuckle H, et al. Effect of mammographic screening from age 40 years on breast cancer mortality (UK Age trial): final results of a randomised, controlled trial. Lancet Oncol. 2020;21(9):1165-1172. Available from: 10.1016/s1470-2045(20)30398-3;https://dx.doi.org/10.1016/ s1470-2045(20)30398-3.

2. Moss SM, Wale C, Smith R, et al. Effect of mammographic screening from age 40 years on breast cancer mortality in the UK Age trial at 17 years' follow-up: a randomised controlled trial. Lancet Oncol. 2015;16(9):1123-1132. Available from: 10.1016/s1470-2045(15)00128-x;https://dx.doi.org/ 10.1016/s1470-2045(15)00128-x.

3. Nelson HD, Fu R, Cantor A, et al. Effectiveness of Breast Cancer Screening: Systematic Review and Meta-analysis to Update the 2009 U.S. Preventive Services Task Force Recommendation. Ann Intern Med. 2016;164(4):244-244. Available from: 10.7326/m15-0969; https://dx.doi.org/10. 7326/m15-0969.

4. Officer PS. Breast cancer screening: Part A. An evidence report to inform an update of the Canadian Task Force on Preventive Health Care; 2011. Available from: https://canadiantaskforce.ca/wp-content/uploads/2019/02/Systematic-Review-Evidence-Report_v2_FINAL.pdf.

5. Gøtzsche PC, Jørgensen KJ. Screening for breast cancer with mammography. Cochrane Database Syst Rev. 2013;(6):1877. Available from: 10.1002/14651858.CD001877.pub5.

6. Keating NL, Pace LE. Breast Cancer Screening in 2018: Time for Shared Decision Making. JAMA. 2018;319(17):1814-1819.

7. Gurreri S, Thériault J, Boucher $\mathrm{S}$. L'indépendance émotionnelle à la mère et au père en lien avec la détresse psychologique chez les jeunes femmes. Psychologie Française. 2019;64:377-390. Available from: 10.1016/j.psfr.2018.01.001;https://dx.doi.org/10.1016/j.psfr.2018.01.001.

8. Doval MB, Volij C, Weisbrot M, et al. Controversias sobre el tamizaje de cáncer mamario con mamografía. Evid Actual Pract Ambul. 2019;22(3):e002023. Available from: http://www.evidencia.org/index.php/Evidencia/article/view/4249.

9. Autier P, Boniol M, Gavin A, et al. Breast cancer mortality in neighbouring European countries with different levels of screening but similar access to treatment: trend analysis of WHO mortality database. BMJ. 2011;343:d4411. Available from: 10.1136/bmj.d4411;https://dx.doi.org/10.1136/bmj. d4411. 
10. Bleyer A, Welch HG. Effect of Three Decades of Screening Mammography on Breast-Cancer Incidence. N Engl J Med. 2012;367(21):1998-2005. Available from: 10.1056/nejmoa1206809;https://dx.doi.org/10.1056/nejmoa1206809.

11. Beral V, Hermon C, Reeves G, et al. Sudden fall in breast cancer death rates in England and Wales. Lancet. 1995;345(8965):1642-1643. Available from: 10.1016/s0140-6736(95)90151-5;https://dx.doi.org/10.1016/s0140-6736(95)90151-5.

12. Park JH, Anderson WF, Gail MH. Improvements in US Breast Cancer Survival and Proportion Explained by Tumor Size and Estrogen-Receptor Status. J Clin Oncol. 2015;33(26):2870-2876. Available from: 10.1200/jco.2014.59.9191;https://dx.doi.org/10.1200/jco.2014.59.9191.

13. Marmot MG, Altman DG, Cameron DA, Independent UK Panel on Breast Cancer Screening, et al. The benefits and harms of breast cancer screening an independent review. Lancet. 2012;380(9855):1778-21786. Available from: 10.1016/S0140-6736(12)61611-0.

14. Di-Sibio AJ. Tamizaje mamográfico del cáncer mama: recomendaciones argentinas. 1st ed. and others, editor. Ciudad Autónoma de Buenos Aires: Instituto Nacional del Cáncer; 2018. Available from: https://www.saludneuquen.gob.ar/wp-content/uploads/2020/09/Ministerio-Salud-Neuqu\%C3\% A9n_Tumores-Efectividad-tamizaje-mamografico-mortalidaden-cm.pdf.

15. Herramienta para decidir sobre el tamizaje de cáncer de mama; 2020. Available from: https://www.fundacionmf.org.ar/visor-producto.php?cod producto $=5603$ [Last access: $2021-02-08]$. 